\title{
Multi-objective of parameters optimization design on Teleoperation Master hand with force feedback
}

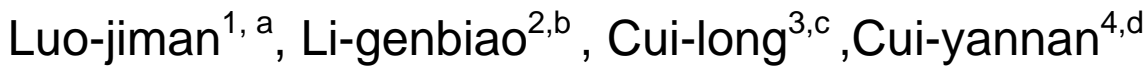 \\ ${ }^{1}$ Shenyang Jianzhu University, Liaoning, Shenyang 110168; \\ ${ }^{2}$ Chinese Academy of Sciences and Shenyang Institute of Automation, Liaoning, Shenyang, \\ 110016; \\ ${ }^{3}$ Shenyang Jianzhu University, Liaoning, Shenyang 110168; \\ ${ }^{4}$ Shenyang Jianzhu University, Liaoning, Shenyang 110168; \\ syljm2006@163.com;664808489@qq.com;cuilong@sia.cn;896910843@qq.com
}

Keywords: Teleoperation master hand with force feedback; Genetic algorithm;Multi-objective parameters optimization design.

\begin{abstract}
Teleoperation master hands with force feedback are used in the project more and more widely and the design requirements about their structure sizes and the scope of work space are also increasing. In order to expand work space of the master hand robot, and to ensure its kinematic accuracy, the structure size parameters of the master hand robot are designed by multi-objective optimization method in the paper, using its work space, output power on its end, as well as precision-weighted inverse solution as optimizing targets. Firstly, the model of parameter optimization is established by taking the radius of upper and lower platform as well as the lengths of the long and short poles of the master hand robot as design variables. Its output power and hinge corner are analyzed as a motion constraints. The objective function of parameter optimization model is based on a weighted form of work space and Jacobi conditions. Then, Model is simulated and calculated by genetic algorithm. The results show that optimized parameters make the scope of work space improved greatly, as well as increasing in accuracy of inverse kinematics and output force.
\end{abstract}

\section{Introduction}

Teleoperation master hand robot development from to now .since its application in the medical field has matured to fatigue resistance and accuracy instead of the person's work. the teleoperation main hand platform driven by the motor transport move under the force feedback control system allows the operator to be able to feel the actual work environment, when faced with a sudden situation can make a reasonable pre-judgment. In addition, their work space, high precision and flexible movement also contributed to its more extensive applications. Thus, such that the resulting master hand to meet the output force to gain the maximum working space through the multi-objective optimization design parameters has very important significance.

Since the robot motion trajectory of space is relatively complex, and genetic algorithms in nonlinear, multi-objective problem without considering too restrictive factors can always seek out the optimal value of the objective function become practicability. Therefore, choosing the genetic algorithm for multi-objective optimization [1],In ensuring the master hand end of the output power to meet the conditions of the situation, seeking to maximize work space. In order to improve the ability of the master hand robot, this paper will be based on the actual engineering specifications and constraints, considering the movement space constraints force feedback and a few other indicators Jacobi conditions, establishing parameters teleoperation master hand optimized design model. Model by genetic algorithm optimization to obtained parameter optimization results[2] . 


\section{Teleoperation master hand agency model}

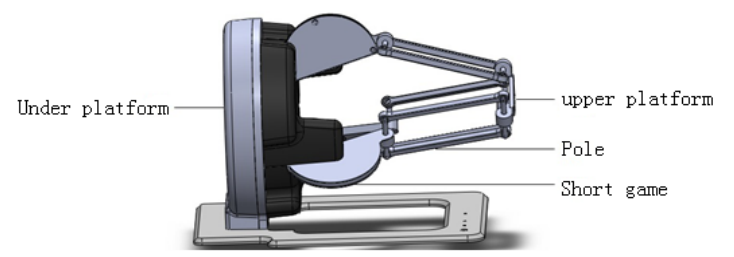

Figure 1 teleoperation master hand agency model

Teleoperation master hand is 3-RUU parallel mechanism has three degrees of freedom of movement. As shown in Figure 1, it is mainly composed of the upper and under platform, as well as the long and short rods. Drive unit mounted on the under platform, driven by a motor hemisphere (short game) rotation pole do planar motion, which led to the platform parallel to the direction move along the $\mathrm{X}, \mathrm{Y}, \mathrm{Z}$ three-axis .

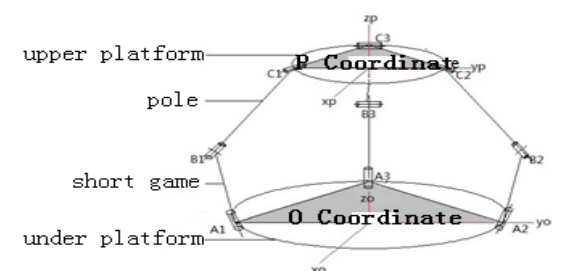

Figure 2 teleoperation master hand configuration diagram

Figure 2 shows the teleoperation master hand configuration diagram that it include upper and lower platforms circumscribed circle and three hinge points, which distributed on the disc uniformly shown as in Figure 1. Building in accordance with Figure 2 coordinate system, it can be determined on the platform three hinge points in the coordinate system of the coordinates of the platform 3 and the lower hinge point coordinate values in the coordinate system coordinate system. The height $\mathrm{H}$ of the platform on the lower platform axially in the same straight line refers to the distance between the two platform [3].The upper and lower platform radii $r, R$, long and short rod length ${ }^{L_{a}}$, ${ }^{L_{b}}$, angle is $^{\beta}$, Where ${ }^{\beta}$ is the angle between the two levers, Requirements $\left(\beta>30^{\circ}\right)$ [3],

$$
H=\sqrt{L a^{2}+L b^{2}+2 L a L b \cos (\beta)-r^{2}+R^{2}-2 r R}
$$

\section{Kinematics and dynamics constraints}

Firstly, the teleoperation master hand attitude and position of the inverse solution will be described below. Because it is consistent depend on three branched chain completely , so only use one of them needs to conduct research and analysis.

Shown in Figure 2, the vectors according to the kinematic equations are:

$$
\begin{aligned}
& \overrightarrow{O A_{i}}+\overrightarrow{A_{i} B_{i}}+\overrightarrow{B_{i} C_{i}}=\overrightarrow{O P}+\overrightarrow{P C_{i}} \\
& L_{i}=\left|l_{i}\right|=\left|\overrightarrow{B_{i} C_{i}}\right|=\left|\overrightarrow{O P}+\overrightarrow{P C_{i}}-\overrightarrow{O A_{i}}-\overrightarrow{A_{i} B_{i}}\right| \quad(i=1,2,3)
\end{aligned}
$$

Where: $l_{i}$ is pole vector for pole vector corresponding scalar, $L_{i}$ namely the long pole; $\overrightarrow{A_{i} B_{i}}$ is short game vector, ${ }^{A_{i} B_{i}}$ is short rod length ${ }^{[4]}$. Because the structure is symmetrical so: $L_{i}=L_{a}, A_{i} B_{i}=L_{b} ; C_{i}$ and $A_{i}$ were ${ }^{i}$ up and down hinge point on the platform in the coordinate system of the coordinates of each body, $P$ and

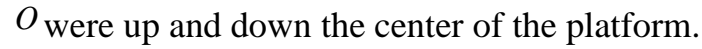

The teleoperation master hand limited by three main constraints during exercise:

(1) the articulation range limits

Inverse kinematics equation can be obtained by being simplified ,

$$
\phi_{i}=2 \arctan \left[z_{p}+\sqrt{z_{p}^{2}+w_{i}^{2}-v_{i}^{2}} /\left(v_{i}+w_{i}\right)\right]
$$

Formula $^{\left(x_{p}, y_{p}, z_{p}\right)}$ based hand end coordinates. $\phi_{i}$ is angle between Short rod and lower platform , ${ }_{i}$ is hinge stop angle. Among them: $a_{1}=90^{\circ}, a_{2}=210^{\circ}, a_{3}=330^{\circ}$. Meet the design requirements under the premise of this article to take $\mathrm{e}^{-90^{\circ}<\theta_{1}<90^{\circ}}$.

Taking $\varphi_{0}=90^{\circ}$ in this article.

(2) output power limit 


$$
\left.\begin{array}{r}
f 1=F * T_{1} \\
\min f 1>f 0
\end{array}\right\}
$$

When the formula ${ }^{f 1}$ is the movement to reach the end of the minimum output power limit position, $F$ is Motor output force, ${ }^{T_{1}}$ is force Jacobian matrix between the upper and lower platform. Taking $f 0=12 N$ in this article.

\section{Parameter optimization of teleoperation master hand}

\subsection{Parameter Optimization Design Model}

Different applications determine the different design parameters of the master hand teleoperation , the paper focuses on the main work space and the end of output power as well as precision-weighted inverse solution in the course of the campaign for multi-objective optimization design goal . Building optimal model by definited design variables and constraints analysis or objective function limitations .

$$
\begin{gathered}
\min : F(R, r, L a, L b)=-k(s) \frac{a b s(\max x(i, j))}{M(i, j)}+k(s+1) \frac{c}{c_{0}} \\
s t: f 1=F^{*} T_{1}
\end{gathered}
$$

Where $a b s(\max x(i, j))(i=1,2,3 ; j=1,2)$ is indicate the scope of the translational platform, Among them $i$ is $\mathrm{X}, \mathrm{Y}, \mathrm{Z}$-axis direction search, 1 tables for $\mathrm{X}$-axis direction, 2 tables for the $\mathrm{Y}$-axis direction, 3tables for $\mathrm{Z}$-axis direction and $j$ represent 1 for $\mathrm{X}, \mathrm{Y}, \mathrm{Z}$ positive; $2 \mathrm{X}, \mathrm{Y}, \mathrm{Z}$ negative. $M(i, j)$ is initial value Before optimization search space boundary, $c_{0}$ is Jacobian condition number before optimization, $C$ is Jacobian condition number after optimization. $k(s)+k(s+1)=1(s=1 \sim 6), k(1) \sim k(7)$ represent an amount corresponding to the respective optimization weight value assigned. According to the initial value and the Jacobian value of the size of the movement space, according to their share some proportion of the initial weights assigned as follows[0.0725;0.0725;0.0725;0.0725;0.3;0.3;0.1];

$f 1$ is minimum output power in Moving boundary, $T_{1}$ is power matrix, $t_{0}$ is initial point, $F$ is motor output power.

$R, r, L_{a}, L_{b}$ as design variables and $s t$ is constraints condition or $F$ as the objective function in optimization Model. Where, the objective function (dimensionless) showed the teleoperation master hand inverse solution in high precision ${ }^{[5]}$ to meet the power constraints and biggest sports space.

Jacobian is Jacobian matrix condition number refers to the ratio of maximum eigenvalues and minimum eigenvalue. In this article.

$$
\text { Jacobin }=-\left[\frac{\partial F}{\partial x}\right]^{-1}\left[\frac{\partial F}{\partial \theta}\right]
$$

Where $F_{i}=\left(x-x_{i}\right)^{2}+\left(y-y_{i}\right)^{2}+\left(z-z_{i}\right)^{2} \quad(i=1,2,3)\left(x_{i}, y_{i}, z_{i}\right)$ is $(x, y, z)$ rotated about its central point, the coordinates of center of the sphere is formed of a sphere of radius. Jacobian Matrix condition number is smaller, the teleoperation master hand robot space more flexible, probability of bizarre lower master hand inverse solution, motion error, the higher the accuracy of inverse kinematics

\subsection{Optimization method}

Genetic algorithm to optimize the design and the amount of each set as follows:

(1)Fitness function: using genetic algorithms to solve the optimization is the minimum of the objective function and therefore use $-(a b s(\max x(i, j))$ to indicate; Fitness $=F(R, r, L a, L b)$;

(2)Population size, select: $M=100$;

(3)The offspring generation, select crossover $p_{c}=0.8$, mutation operator $p_{a}=0.01$;

(4)Offspring termination condition evaluation: If you use function Fitness change over the previous generation function is less than the $0.01 \%$ default iteration terminates with a maximum output of individual fitness as an optimal output, the ${ }^{\sum(a b s(\max x(i, j))}$ size of the value as a result of the optimization evaluation.

\section{3 optimization results}

Before optimization model for the initial values of the parameters as shown in Table 1: 


\begin{tabular}{cc} 
Table 1 before optimization parameters \\
\hline$(R, r, L a, L b)=(0.075,0.037,0.146,0.037)$ Units is $\mathrm{m}$ \\
\hline$\sum(a b s(\max x(i, j))$ & 0.68 \\
$C$ & 11.448 \\
$f 1$ & $13.985 \mathrm{~N}$ \\
$t_{0}$ & $(0,0,-0.1138)$ \\
Fitness & -0.12
\end{tabular}

The values in Table 1 are substituted into the objective function and use of genetic algorithm optimization to simulation and test results.

The resulting value of design variables to optimize the extraction of four sets of parameters by iterative model ultimately converge are shown in Table 2.

Group 4 design variables optimized set of values in Table 2

\begin{tabular}{cccc}
\hline$R$ & $r$ & $L a$ & $L b$ \\
\hline 0.112 & 0.037 & 0.209 & 0.100 \\
0.115 & 0.065 & 0.215 & 0.104 \\
0.117 & 0.090 & 0.212 & 0.106 \\
0.116 & 0.069 & 0.233 & 0.110
\end{tabular}

After optimization of the extraction of the four sets of values are averaged, and its size is: $(R, r, L a, L b)=(0.115,0.065,0.215,0.104)$. Results combining the optimized objective function calculation and validation. Each parameter value obtained after optimization, the results shown in Table 3.

Each parameter value optimization in Table 3

\begin{tabular}{cc}
\hline$(R, r, L a, L b)=(0.115,0.065,0.215,0.104)$ \\
\hline$\sum(a b s(\max x(i, j))$ & 1.12 \\
$C$ & 1.95 \\
$f 1$ & $16.98 \mathrm{~N}$
\end{tabular}

Fitness $-1.21$

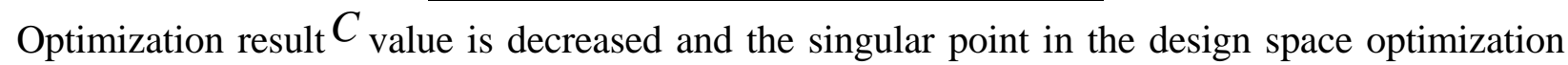
of Jacobian matrices do not exist, the accuracy of the inverse solution of the inverse kinematics

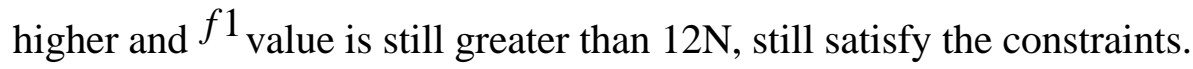

Now compare the before and after simulation to optimize both extreme positions minimum output power and motion space analysis to determine whether it is feasible to optimize the results.

1, the end of the output power to optimize conditions:

(1) Comparison of the output power before and after optimization

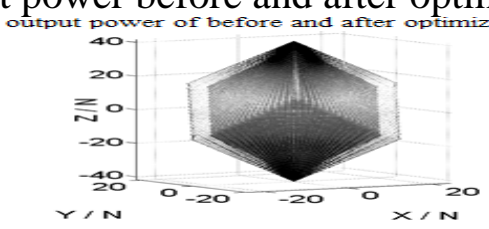

Figure 3 output power of before and after optimization

Figure 2 the translucent portion is after optimize out power and black part is before optimization out power. It can be seen as a whole relative before the optimized expanded a lot .After optimization of the output value of the boundary description of the minimum force than before is expanding to meet the restrictions force constraints.

In order to better observe the contrast, now making before and after optimize contrast: 


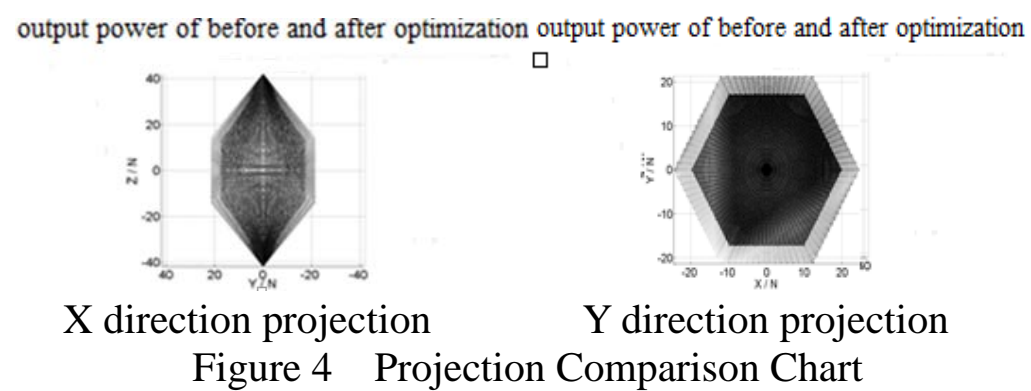

Large gray area in Figure 3 is a partial projection optimized and the black part is projection before optimization.It will optimize the $\mathrm{X}, \mathrm{Y}, \mathrm{Z}$ axis force on the boundary before and after contrast and can be accurately optimized contrast in extreme position $\mathrm{X}, \mathrm{Y}, \mathrm{Z}$ axis is greater than optimization before the data with a projection on the two coordinate axis direction,Specific value is increased from the previous $13.985 \mathrm{~N}$ to $16.98 \mathrm{~N}$, much greater than $12 \mathrm{~N}$, it is explain that the constraint condition is satisfied described.

2, the working space optimization condition

According to the results of the genetic algorithm optimization compared before and after optimization workspace. As figure 4:

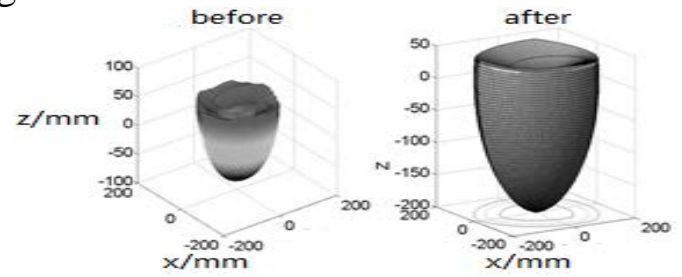

Workspace of before and after optimization in Figure 5

From figure 4 can be obtained after optimization $\mathrm{X}, \mathrm{Y}, \mathrm{Z}$ coordinate value raise a lot than the initial value, now moving space projected for a more intuitive comparison.

Firstly, projection in the Z-axis direction and the result is displayed as in the $\mathrm{X}, \mathrm{Y}$ axis direction translational space.

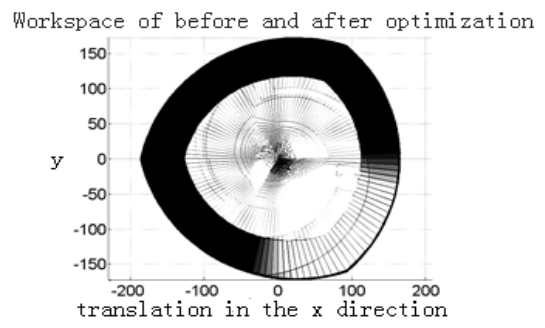

Figure $6 \quad \mathrm{X}, \mathrm{Y}$ movable parallel to the direction of space / $\mathrm{mm}$

Fig inner contour portion of the white space is before optimization and the outer contour of the black area is the space optimized, compared to optimized the space-axis direction of $\mathrm{X}, \mathrm{Y}$, significantly increases, about 1.64 times compare to before.

Secondly, projection in the $\mathrm{X}$-axis direction and the result is displayed as in the $\mathrm{Y}, \mathrm{Z}$ axis direction translational space.

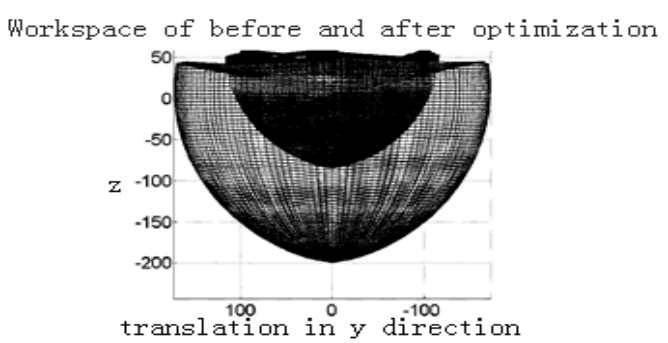

Figure $7 \mathrm{Y}, \mathrm{Z}$ movable parallel to the direction of space / $\mathrm{mm}$

Due to the optimization coordinate value of the initial point $(0,0,-113.8)$ unit is $\mathrm{mm}$, so the value of the positive and negative direction of the $\mathrm{Z}$ axis in both negative, controlled observation shown as in Table 4 and Figure 7, except in the positive direction of the Z-axis from the previous -73.80 decreased to -93.8 , the rest of the data values for each search direction has obviously improved, 
especially in the negative direction of the Z-axis by -313.8 -203.8 before upgrading, twice than before (calculated from the initial point). The results showed that the actual workspace optimized on the X, Y, Z-axis direction has been greatly improved, the space corresponding evaluation index value increased from 0.681 .12 , about 1.66 times than before . Optimization results are very satisfactory, so optimization is feasible.

\section{Conclusion}

The mechanism parameters optimization problem of teleoperation master hand is studied in this paper, by establishing an integrated optimization model about workspace, multi-objective parameter constraints force and motion inverse solution accuracy as the objective function, and using genetic algorithms to optimize the design parameters to solve.

In the case of work to meet the actual needs, the result will be optimized comparison with prior and the following results were obtained: End extreme positions minimum output power increase from the previous $13.985 \mathrm{~N}$ to $16.98 \mathrm{~N}$. Boundary value workspace only slightly reduced in the $\mathrm{Z}$ direction, the direction of the rest of the boundary value is about 1.6 times than before. The results show that under the restrictions satisfy the constraints, the workspace optimized been significantly improved so that the actual work capability of the teleoperation master hand has been improved greatly, the study provides an effective and feasible way for teleoperation master hand design and it is very meaningful.

\section{References}

[1] Caoxiaoning.speed train bogie test rig 3- DOF platform kinematics and working space research [J],.Jilin University doctoral dissertation, 2013.

[2] Wanggengxang,Yuandaning.Space 4-SPS / CU parallel kinematics former transactions analyzed [J].Agricultural Machinery, 2012.43 (3): 207-212,199.

[3] Renrui Key technology platform of 6DOF any attitude control, China Research Institute of Engineering Physics PhD thesis, 2012.8.10.

[4] Kubler L,Henninger C,Eberhard P.Multi-criteria optimization of Hexapod Machine [J]. Mutibody system Dy-namics,2005,14:225-250.

[5] Faas Daniela,Fischer, vance Judy M.Interactive Mesh-Free Stress Analysis for Mechanical Design Assembly With Haptics.ASME 2007Interactional Design Engineering Technical Conference and Computer And Information in Engineering Conference.2008:1359 1365. 